\title{
ДИНАМИКА УРОВНЕЙ ГОРМОНОВ РЕНИН-АНГИОТЕНЗИНОВОЙ СИСТЕМЫ \\ ПОЧКИ И КОРЫ НАДПОЧЕЧНИКОВ У БОЛЬНЫХ ПРОСТЫМИ СОЛИТАРНЫМИ КИСТАМИ ПОЧКИ, ОСЛОЖНЕННЫМИ СИМПТОМАТИЧЕСКОЙ АРТЕРИАЛЬНОЙ ГИПЕРТЕНЗИЕЙ
}

\author{
А.А. Черенков, В.Г. Аристархов
}

ГБУРО «Городская клиническая больница №11» г. Рязань

ГБОУ ВПО «Рязанский государственный медицинский университет имени академика

И.П. Павлова» Министерства здравоохранения и социального развития РФ, г. Рязань

В статье приводятся результаты хирургической коррекции симптоматической артериальной гипертензии у 95 пациентов, страдающих простыми солитарными кистами почечной паренхимы. У данных больных проведено исследование уровней гормонов ренина, альдостерона, кортизола в сопоставлении с динамикой цифр систолического и диастолического артериального давления. На основании анализа полученных в ходе исследования данных высказывается гипотеза о зависимости механизма формирования артериальной гипертензии от топографии простой кисты почки.

Ключевые слова: почка, кора надпочечников, ренин-ангиотензиновая система, оценка, гормоны, симптоматическая артериальная гипертензия.

Простые кисты почек являются распространенной патологией верхних мочевых путей, требующей хирургической коррекции. Кистозные поражения почек встречаются с частотой $3 \%$ среди пациентов урологического и нефрологического профиля [4], а в общей популяции у 12\% людей имеются неосложненные кисты почек [2].

Наиболее часто простая киста почки не имеет клинических проявлений и выявляется случайно. В других случаях кистозное образование почки обусловливает развитие болевого синдрома, гематурии, уростаза в верхних мочевых путях и симптоматической артериальной гипертензии (АГ) [3,6].

Симптоматическая АГ в качестве клинического симптома простой кисты почки наблюдается в 20\% случаев и является показанием к хирургическому устранению простой кисты почки [7,8,9].

Изучение механизмов развития и методов хирургической коррекции АГ является актуальной медицинской проблемой, так как формирующаяся под воздействием кистозного образования симптоматическая ренальная АГ плохо поддается медикаментозному лечению и часто протекает злокачественно: вызывает ишемическую болезнь сердца, инфаркт миокарда, мозговые инсульты, сердечную и почечную недостаточность [6].

\section{Материалы и методы}

Исследование основывается на результатах анализа протоколов наблюдения 95 пациентов, страдающих солитарными простыми кистами почечной паренхимы. Клинически простая киста почки во всех наблюдениях проявляется в виде симптоматической АГ: у 59 пациентов повышенные цифры АД являются единственным симптомом, ассоциированным с простой кисты почки, а у 36 больных имеет место сочетание АГ с другими проявлениями кистозных заболеваний почек. Средний возраст больных составил $45,1 \pm 6,42$ года. Половой со- 
Российский медико-биологический вестник имени академика И.П. Павлова, №2, 2012 г.

став больных следующий: мужчин - 44, женщин - 51 пациент.

Пациенты, вошедшие в исследование, разделены на 3 группы наблюдения в зависимости от топографии простой кисты почки. I группу наблюдения составили 23 больных солитарными кистами верхнего сегмента почки; во II группу вошли 35 пациентов с кистами среднего сегмента почки, в 37 наблюдениях простая киста почки была локализована в нижнем полюсе почки (III группа наблюдения).

Целью подобного разделения пациентов на 3 группы наблюдения является клинический анализ зависимости топографии простой кисты почки на развитие АГ ренального генеза у данной когорты больных.

Все пациенты $(\mathrm{n}=95)$ перенесли хирургическое лечение по поводу кистозного поражения почки со стойким безрецидивным эффектом. Основным критерием эффективности проведенного хирургического лечения в плане коррекции АГ у названных 95 больных было снижение цифр артериального давления (АД) минимум на 10 мм рт.ст. либо возвращение показателя АД к нормальным значениям (норма ВО3, 1999: АД систолическое не выше 140 мм рт.ст.; АД диастолическое не более 90 мм рт.ст).

Всем пациентам ( $\mathrm{n}=95)$ проводилось исследование уровней гормонов кортизола, альдостерона, ренина и мониторинг АД: до оперативного лечения, во время операции (либо в ранний послеоперационный период - не позднее двух часов с момента операции), через 6 месяцев и через год после хирургического лечения простой кисты почки. Полученные данные сопоставлялись с топографией кистозного образования.

\section{Результаты и их обсуждение}

Достигнуть коррекции симптоматической АГ согласно принятым в эксперименте эквивалентам эффективности после проведенного хирургического лечения удалось у 54 пациентов $(56,8 \%)$.

Среди пациентов I группы наблюдения эффективное в плане коррекции симптоматической АГ оперативное лечение наблюдалось в 9 случаях. Динамика уровней изучаемых гормонов и АД данных больных приведены в таблице 1 .

Таблица1

Динамика значений уровня гормонов ренина, альдостерона, кортизола и АД у больных кистами верхнего полюса почки, хирургическое лечение у которых привело к снижению цифр АД $(n=9)$

\begin{tabular}{|l|c|c|c|c|}
\hline & До лечения & Во время операции & Через 6 месяцев & Через год \\
\hline Кортизол (нмоль/л) & $467,1 \pm 12,1$ & $\begin{array}{c}494,7 \pm 10,3 \\
(+5,6 \%)\end{array}$ & $\begin{array}{c}388,1 \pm 11,1 \\
(-16,9 \%)\end{array}$ & $\begin{array}{c}309,6 \pm 18,9 \\
(-33,7 \%)\end{array}$ \\
\hline Альдостерон (пг/мл) & $181,9 \pm 10,1$ & $\begin{array}{c}208 \pm 12,4 \\
(+12,5 \%)\end{array}$ & $\begin{array}{c}165,4 \pm 11,8 \\
(-9,1 \%)\end{array}$ & $\begin{array}{c}133,1 \pm 20,8 \\
(-26,8 \%)\end{array}$ \\
\hline Ренин (нг/мл/час) & $0,7 \pm 0,12$ & $\begin{array}{c}0,71 \pm 0,14 \\
(+2,2 \%)\end{array}$ & $\begin{array}{c}0,51 \pm 0,09 \\
(-27,1 \%)\end{array}$ & $\begin{array}{c}0,54 \pm 0,2 \\
(-22,9 \%)\end{array}$ \\
\hline $\begin{array}{l}\text { АД систолическое } \\
\text { (мм.рт.ст.) }\end{array}$ & $149,3 \pm 4,3$ & $\begin{array}{c}151,9 \pm 4,1 \\
(+1,4 \%)\end{array}$ & $\begin{array}{c}127 \pm 3,7 \\
(-14,9 \%)\end{array}$ & $\begin{array}{c}128,5 \pm 3,5 \\
(-13,9 \%)\end{array}$ \\
\hline $\begin{array}{l}\text { АД диастолическое } \\
\text { мм.рт.ст.) }\end{array}$ & $102,1 \pm 1,7$ & $\begin{array}{c}102,7 \pm 1,79 \\
(+0,6 \%)\end{array}$ & $\begin{array}{c}86,1 \pm 1,1 \\
(-15,7 \%)\end{array}$ & $\begin{array}{c}88,5 \pm 0,9 \\
(-13,3 \%)\end{array}$ \\
\hline
\end{tabular}

Как видно из табл. 1 средние значения альдостерона превышают уровни нормы $(10$ - 105 пкг/мл). Среднее значение уровня кортизола крови расценивается как высокое и приближается к верхнему порогу референсных значений уровня данного гормона $(250$ - 650 нмоль/л).

Хирургическое лечение простой кисты почки у изучаемых больных способствовало выраженному снижению уровней гормонов коры надпочечника в отдаленном послеоперационном периоде: 
уровень кортизола снизился на 33,7\%, значения альдостерона регрессировали на $26,8 \%$. На этом фоне отмечается снижение цифр АД систолического на $13,9 \%$ и АД диастолического на $13,3 \%$.

Одновременно с этим, отмечается редукция значений уровня ренина плазмы крови: на 27,1\% через 6 месяцев после хирургического лечения и на $22,9 \%$ через год после операции. При этом верхний порог среднего уровня ренина не превышает нормальных значений (в покое: 0,5-1,9 нг/мл/час).
При сопоставлении полученных данных с топографией простой кисты почки выясняется, что у 9 пациентов, перенесших эффективное в плане коррекции АГ хирургическое лечение, жидкостное образование было локализовано по передней поверхности верхнего сегмента почки и немного медиальнее (рисунок 1б). Подобная топография простой кисты почки обуславливает наиболее интимный контакт кисты со структурами надпочечника и вызывает компрессию этой железы [5].

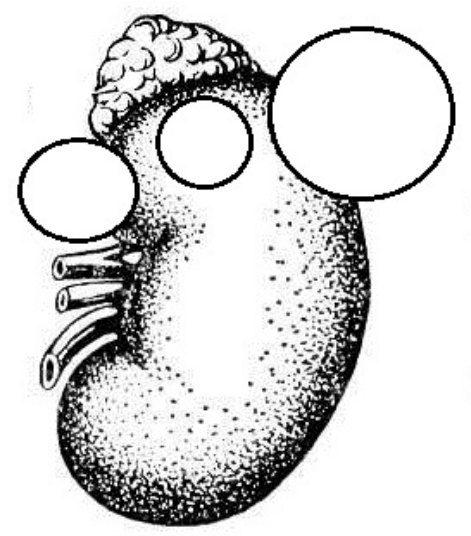

A

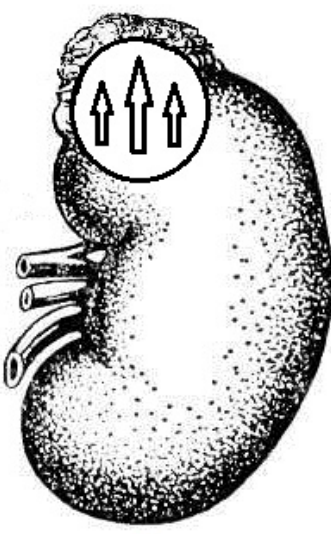

Б

Рис. 1 Схема патогенеза АГ при локализации кисты в верхнем полюсе почки: $\mathbf{a}$ - отсутствие воздействия жидкостного образования на структуры надпочечника; б - компрессионное воздействие кистозного образования на анатомические элементы надпочечника

У остальных 14 пациентов перенесших неэффективное в плане коррекции АГ хирургическое лечение уровни изучаемых гормонов не выходят за пределы нормальных значений, а топография простой кисты почки, согласно данным дополнительных методов исследования, исключает интимный контакт кисты и надпочечника.

Сопоставление динамики уровней кортизола и альдостерона плазмы крови с динамикой АД и топографией простой кисты почки в отсутствие превышения уровнем ренина плазмы крови значений нормы указывает на существенную роль в формировании АГ при простых кистах верхнего полюса почки вовлечения в патологический процесс анатомических элементов надпочечника.
Среди пациентов II группы наблюдения эффективность хирургического лечения в виде снижения цифр АД систолического. минимум на 10 мм.рт.ст либо нормализация АД была достигнута у 21 больного (60\%).

Динамика уровней изучаемых гормонов пациентов II группы наблюдения, хирургическое лечение в плане коррекции АГ у которых расценивается как эффективное представлены в таблице 2.

Как видно из табл. 2 у 21 пациента II группы наблюдения отмечается превышение уровнем ренина плазмы крови верхнего порога нормы на $17,7 \%$. В отдаленном послеоперационном периоде отмечается значительное снижение среднего уровня ренина плазмы крови: на $64.5 \%$ 
Российский медико-биологический вестник имени академика И.П. Павлова, №2, 2012 г.

Таблица 2

\begin{abstract}
Динамика уровня ренина, альдостерона плазмы крови в сопоставлении со значениями цифр АД больных кистами среднего сегмента почки, у которых хирургическое лечение в плане коррекции АГ расценивается как эффективное (n=21, II группа наблюдения)
\end{abstract}

\begin{tabular}{|c|c|c|c|c|}
\hline & До лечения & Во время операции & Через 6 месяцев & Через год \\
\hline Кортизол (нмоль/л) & $314,2 \pm 21,7$ & $\begin{array}{c}321,4 \pm 17,8 \\
(+2,2 \%)\end{array}$ & $\begin{array}{c}316,1 \pm 20,2 \\
(+0,6 \%)\end{array}$ & $\begin{array}{c}313,5 \pm 21,2 \\
(-0,2 \%)\end{array}$ \\
\hline $\begin{array}{l}\text { Альдостерон } \\
\text { (пг/мл) }\end{array}$ & $234,7 \pm 31,3$ & $\begin{array}{c}244,5 \pm 34,9 \\
(+4 \%)\end{array}$ & $\begin{array}{c}142,1 \pm 23,7 \\
(-39,5 \%)\end{array}$ & $\begin{array}{c}143,3 \pm 22,8 \\
(-38,9 \%)\end{array}$ \\
\hline Ренин (нг/мл/час) & $2,31 \pm 0,43$ & $\begin{array}{c}2,33 \pm 0,49 \\
(+0,9 \%)\end{array}$ & $\begin{array}{c}0,82 \pm 0,17 \\
(-64,5 \%)\end{array}$ & $\begin{array}{c}0,77 \pm 0,18 \\
(-66,7 \%)\end{array}$ \\
\hline $\begin{array}{l}\text { АД систолическое } \\
\text { (мм.рт.ст.) }\end{array}$ & $151,2 \pm 4,5$ & $\begin{array}{c}155,1 \pm 4,1 \\
(+2,5 \%)\end{array}$ & $\begin{array}{c}130,2 \pm 3,5 \\
(-13,9 \%)\end{array}$ & $\begin{array}{c}129,2 \pm 3,3 \\
(-14,6 \%)\end{array}$ \\
\hline $\begin{array}{l}\text { АД диастолическое } \\
\text { (мм.рт.ст.) }\end{array}$ & $103,4 \pm 1$ & $\begin{array}{c}105,2 \pm 1,1 \\
(+1,7 \%)\end{array}$ & $\begin{array}{c}87 \pm 2,9 \\
(-15,9 \%)\end{array}$ & $\begin{array}{l}88,1 \pm 3,6 \\
(-14,8 \%)\end{array}$ \\
\hline
\end{tabular}

через 6 месяцев после хирургического лечения и на $66,7 \%$ через год после операции. Одновременно с этим происходит редукция средних значений уровня альдостерона плазмы крови на $38,9 \%$, что объясняется наличием в регуляции уровня альдостерона прямой зависимости от уровня ренина плазмы крови [1].

Динамика уровня кортизола плазмы крови за время наблюдения пациентов II группы показывает незначительные колебания значений данного гормона относительно первоначального уровня, что исключает вовлечение в патологический процесс анатомических структур надпочечника.

При сопоставлении полученных результатов с топографией простой кисты почки выясняется, что у 19 из названных пациентов простая киста почки была расположена окололоханочно, а в 2 наблюдениях кистозное образование было локализовано интрапаренхиматозно по медиальной поверхности почки.

Подобная топография жидкостных образований почки обуславливает высокий риск компрессионного воздействия кисты на структурные элементы почечных ворот, что может приводить к развитию уродинамических нарушений пассажа мочи на стороне патологического процесса и поддержанию хронического воспалительного процесса почечной паренхимы. Хронический пиелонефрит, в свою очередь, усугубляет ишемические изменения паренхимы почки и, таким образом, усугубляет выраженность симптоматической АГ.

Подобные уродинамические изменения прослеживаются, по данным дополнительных методов исследования, у 11 больных II группы наблюдения, что выражается в расширении лоханки почки на стороне простой кисты от 12 до 18 мм $(\mathrm{Mcp}=13,3 \pm 1,7 \mathrm{Mм})$ по данным УЗИ почек. Анамнестические данные выявляют наличие хронического пиелонефрита у 7 названных пациентов.

Результаты мониторинга уровней исследуемых гормонов у пациентов (n $=14$ ), перенесших неэффективное в плане коррекции гипертензии лечение указывает на отсутствие превышения среднего уровня ренина, альдостерона верхних порогов референсных значений. Простая киста почки у данных больных располагалась более латерально относительно ворот почки, что исключало компрессию кистой полостной системы почки.

Результаты мониторинга уровней изучаемых гормонов плазмы крови указывает на ведущую роль ренинангиотензиновой системы почки в формировании симптоматической АГ при локализации кисты в среднем сегменте почки Результаты сопоставления уровней изучаемых гормонов, артериального давления с топографией простой кисты почки 
Российский медико-биологический вестник имени академика И.П. Павлова, №2, 2012 г.

указывает на преимущественное формирование симптоматической гипертензии по паренхиматозному механизму. Определенную роль в формировании артериальной гипертензии при локализации кисты в среднем сегменте почки имеют нарушения адекватного пассажа мочи на стороне простой кисты почки, обусловливающие хронический воспалительный процесс паренхимы почки и усугубляю- щие ишемические изменения последней.

Простые кисты нижнего полюса почки были наиболее значительных размеров (средний размер 8,3 $\pm 1,43$ см, с диапазоном значений от 4,5 см до $12,5 \mathrm{~cm})$.

Результаты мониторинга уровней исследуемых гормонов и артериального давления у 37 пациентов III группы наблюдения приведены в таблице 3 .

Таблица 3

Динамика значений уровня гормонов ренина, альдостерона, кортизола и АД у пациентов, страдающих кистозными поражениями нижнего полюса почки, осложненными АГ (группа III n = 37)

\begin{tabular}{|l|c|c|c|c|}
\hline & До лечения & Во время операции & Через 6 месяцев & Через год \\
\hline Кортизол (нмоль/л) & $302,1 \pm 23,1$ & $\begin{array}{c}311,4 \pm 21,8 \\
(+3 \%)\end{array}$ & $\begin{array}{c}310,1 \pm 23,1 \\
(+2,6 \%)\end{array}$ & $\begin{array}{c}309,6 \pm 23,7 \\
(+2,4 \%)\end{array}$ \\
\hline Альдостерон (пг/мл) & $202,7 \pm 27,3$ & $\begin{array}{c}217,2 \pm 31,2 \\
(+7,1 \%)\end{array}$ & $\begin{array}{c}148,1 \pm 15,1 \\
(-26,9 \%)\end{array}$ & $\begin{array}{c}140,9 \pm 14,8 \\
(-30,5 \%)\end{array}$ \\
\hline Ренин (нг/мл/час) & $1,88 \pm 0,39$ & $\begin{array}{c}1,89 \pm 0,4 \\
(+0,5 \%)\end{array}$ & $\begin{array}{c}0,83 \pm 0,13 \\
(-55,8 \%)\end{array}$ & $\begin{array}{c}0,77 \pm 0,1 \\
(-59 \%)\end{array}$ \\
\hline $\begin{array}{l}\text { АД систолическое } \\
\text { (мм.рт.ст.) }\end{array}$ & $146,1 \pm 4,3$ & $\begin{array}{c}154,2 \pm 4,2 \\
(+5,3 \%)\end{array}$ & $\begin{array}{c}137,2 \pm 5,7 \\
(-6,1 \%)\end{array}$ & $\begin{array}{c}131,1 \pm 6,8 \\
(-10,3 \%)\end{array}$ \\
\hline $\begin{array}{l}\text { АД диастолическое } \\
\text { (мм.рт.ст.) }\end{array}$ & $99,8 \pm 1,9$ & $\begin{array}{c}103,3 \pm 2,3 \\
(+3,4 \%)\end{array}$ & $\begin{array}{c}93,5 \pm 4,3 \\
(-6,3 \%)\end{array}$ & $\begin{array}{c}90,1 \pm 5,3 \\
(-9,7 \%)\end{array}$ \\
\hline
\end{tabular}

Результаты мониторинга уровней гормонов и АД у пациентов III группы наблюдения указывают на ведущее место в формировании симптоматической АГ при подобной локализации кисты ренинангиотензиновой системы почки, что проявляется в снижении уровней гормонов ренина и альдостерона на 59\% и $30,5 \%$ соответственно.

Анализ зависимости уровня ренина плазмы крови от размеров кистозного образования почки указывает на прямую положительную корреляционную взаимосвязь $(\mathrm{r}=+0,82)$ двух заданных показателей, что показывает преимущественное развитие ишемических процессов в почечной паренхиме по паренхиматозному типу. Клинически данные факты проявляются в коррекции АГ у 24 пациентов.

Среди 13 пациентов, перенесших неудачное в плане коррекции АГ хирургическое лечение, значения уровеня ренина не превышают пределов нормы; средний диаметр кистозного образования составил $5,9 \pm 0,8$ см, что в значительной степени меньше средних размеров простой кисты почки $(9,6 \pm 1$ см) у пациентов $(\mathrm{n}=24)$, оперативное лечение у которых привело к снижению АД согласно определяющим критериям; нарушения адекватного пассажа мочи в исследуемых 13 случаях по данным дополнительных методов исследования не отмечается.

\section{Выводы}

Суммируя данные анализа зависимости топографии кистозного образования почки на формирование симптоматической ренальной гипертензии, можно сделать вывод, что от локализации простой кисты почки зависят патогенетические аспекты формирования симптоматической АГ. Так, симптоматическая АГ при локализации кисты в верхнем полюсе почки в определенной степени обусловлена участием в патологическом процессе анатомических 
структур надпочечника, что проявляется повышением уровней вазопрессорных гормонов коры данной железы. Простые кисты среднего сегмента почки вызывают развитие симптоматической гипертензии по паренхиматозному типу посредством активации вазоактивных гормонов ренинангиотензиновой системы почки. При окололоханочном расположении простой кисты почки либо крупных размерах, кисты среднего сегмента почки нередко вызывают компрессию анатомических элементов полостной системы почки, что приводит к развитию хронического пиелонефрита и усилению ишемии почечной паренхимы, что является дополнительным фактором в развитии АГ. Кистозные поражения нижнего полюса почки, являясь наиболее крупными по размеру, обуславливают формирование артериальной гипертензии посредством компрессионного давления кисты на нормальную паренхиму почки с развитием ишемии последней и симптоматической АГ.

Изучение уровней гормонов ренина, ангиотензина, альдостерона в сопоставлении с локализацией и размером простой кисты почки, дополненное в ряде случаев данными дополнительных методов исследования, позволяют провести четкую дифференциальную диагностику между симптоматической артериальной гипертензией и эссеницальной гипертензией (гипертонической болезнью), что позволит избежать в ряде случаев неэффективного в плане коррекции артериальной гипертензии инвазивного хирургического лечения.

\section{Литература}

1. Алмазов В.А. Артериальная гипертензия и почки / В.А. Алмазов, Е.В. Шляхто. - СПб.: Изд-во СПбГМУ им. акад. И.П. Павлова. - СПб., 1999. - 296 с.

2. Аляев Ю.Г. Диагностика и лечение жидкостных образований почек и забрюшинного пространства / Ю.Г. Аляев, В.А. Григорян, Т.Г. Маркосян. Смоленск: Маджента, 2007. - 160 с.

3. Комяков Б.К. Артериальная гипертензия, вызванная кистой почки небольших размеров / Б.К. Комяков, Б.Г. Гулиев // Урология. - 2009. - №1. - С. 66-67.

4. Лопаткин Н.А. Простая киста почек / Н.А. Лопаткин, Е.Б. Мазо. - М.: Медицина, 1982. - 140 с.

5. Салынов А.В. Причина и профилактика артериальной гипертензии у больных нефролитиазом после дистанционной литотрипсии: дис... канд. мед. наук / А.В. Салынов. - Рязань, 2006. - 138 с.

6. Соловьева А.В. Симптоматические артериальные гипертензии при болезнях почек / А.В. Соловьева // Сочетанная патология в клинической практике: сборник научных трудов, посвященный юбилею Заслуженного деятеля науки РФ проф. Гармаша В.Я. - Рязань, 2011. - С. 10-11

7. Лапароскопическое иссечение кист почек / Ю.В. Иванов [и др.] // Эндоскопическая хирургия. - 2009. - №3. - С. 15-19.

8. Is it safe and effective to treat complex renal cysts by the laparoscopic approach? / T. Pinheiro [et al.] // Endourological Society. - 2011. - Vol.25, №3. - P.471476.

9. Steg A. Les affections kystiques du rein de 1 adulte 65-e section: AFU Paris. Oct. 1975 // J. U. Nephrol. - 1975. - Vol. 81. - P. 21. 


\title{
THE DYNAMICS OF ADRENAL CORTEX AND RENAL RENIN-ANGIOTENSIN SYSTEM HORMONE LEVEL OF THE PATIENTS WITH SIMPLE SOLITARY CYSTS, COMPLICATED BY SYMPTOMATIC ARTERIAL HYPERTENSION
}

\author{
A.A. Cherenkov, V.G. Aristarkhov
}

The article deals with the results of the symptomatic arterial hypertension surgical correction of 95 patients, suffering simple solitary renal parenchyma cysts. These patients were examined for hormone levels of renin, aldosterone and cortisol versus figure dynamics of systolic and diastolic arterial tension. A hypothesis about a dependence of arterial hypertension forming mechanism on simple renal cyst topography is formulated on the basis of data analysed.

Key words: kidney, adrenal cortex, the renin-angiotensin system, evaluation, hormones, symptomatic hypertension.

Черенков Андрей Аркадьевич.

г. Рязань, ул. Новоселов, д. 26/17.

Тел.: 41-30-12.

E-mail: Cherenkov_62@BK.ru. 